\title{
Heavy top limit and double-logarithmic contributions to Higgs production at $m_{H}^{2} / s \ll 1$
}

\author{
F. Hautmann ${ }^{\mathrm{a}, \mathrm{b}}$ \\ a Physics Department, University of Oregon, Eugene, OR 97403, USA \\ b Institut für Theoretische Physik, Universität Regensburg, D-93040 Regensburg, Germany
}

Received 20 March 2002; accepted 13 April 2002

Editor: H. Georgi

\begin{abstract}
Next-to-next-to-leading order (NNLO) QCD corrections to Higgs boson hadroproduction have recently been calculated in the heavy top-quark limit $m_{t} \rightarrow \infty$. The $m_{t} \rightarrow \infty$ limit introduces double-logarithmic corrections in $\ln x$, with $x \equiv m_{H}^{2} / s$. We identify these corrections order by order in $\alpha_{s}$. As an application, we derive an analytic expression for the dominant $x \ll 1$ part of the NNLO coefficient. (C) 2002 Elsevier Science B.V. All rights reserved.
\end{abstract}

Recently, Harlander and Kilgore [1] have completed the calculation of the next-to-next-to-leading order (NNLO) QCD correction to Higgs boson production in hadron-hadron collisions. The calculation is done in the heavy top quark limit, in which $m_{t} \rightarrow \infty$ and all other quark masses vanish. In this limit, the effective Lagrangian of [2] is used for coupling the Higgs boson to gluons. The hard scattering coefficient expanded through NNLO has the structure

$$
\begin{aligned}
C\left(\alpha_{s}, x, m_{H}^{2} / \mu^{2}\right) \\
=c^{(0)}(x)+\frac{\alpha_{s}}{\pi}\left[c^{(1)}(x)+\bar{c}^{(1)}(x) L\right] \\
\quad+\left(\frac{\alpha_{s}}{\pi}\right)^{2}\left[c^{(2)}(x)+\bar{c}^{(2)}(x) L+\overline{\bar{c}}^{(2)}(x) L^{2}\right] \\
\quad+\cdots,
\end{aligned}
$$

E-mail address: hautmann@neutrino.uoregon.edu (F. Hautmann).

where $L \equiv \ln \left(m_{H}^{2} / \mu^{2}\right)$, with $m_{H}$ the Higgs boson mass and $\mu$ the factorization scale; $x=m_{H}^{2} / s$, with $s$ the center-of-mass energy. The term in $c^{(0)}$ is the leading-order term [3]; the term in $c^{(1)}, \bar{c}^{(1)}$ is the nextto-leading-order term [4].

Ref. [1] presents the result for $c^{(2)}$ as an expansion in powers of $(1-x), x=1$ being the production threshold. Explicit numerical results are given up to a very high order in this expansion, $(1-x)^{16}$. Once the hard scattering coefficient is convoluted with the parton densities, this expansion leads to a corresponding expansion for the cross section. This is demonstrated numerically to converge well [1]. As discussed by Catani, de Florian and Grazzini [5] and by Harlander and Kilgore [1], the reason for the convergence of the $(1-x)$ expansion is mainly kinematical, and depends on the steeply rising behavior of parton luminosities for decreasing momentum fractions. This behavior also explains why results based only on 
soft [6,7] and virtual [8] contributions give a reasonably good approximation to the full answer.

In this Letter we focus on the influence of the $m_{t} \rightarrow$ $\infty$ approximation on the $x \ll 1$ part of the coefficient. It was observed in [9] that a local Higgs-gluon coupling gives rise to double-logarithmic corrections in $\ln x$. In this note we identify these corrections explicitly order by order in $\alpha_{s}$. In particular we obtain an analytic result for the dominant $x \ll 1$ part of the NNLO coefficient.

Although the $x \ll 1$ behavior is unlikely to affect the convergence of the inclusive cross section at NNLO, it may however affect distributions associated to less inclusive observables. This analysis may thus be useful for further evaluations of the hard scattering.

Let us consider the $x \ll 1$ corrections in the framework of the effective Lagrangian [2] for the Higgs boson coupling to gluons:

$\mathcal{L}_{\text {eff }}=\left(G_{F} \sqrt{2}\right)^{1 / 2} K\left(\alpha_{s}\right) G_{\mu \nu}^{a} G^{a \mu \nu} H$,

where $K\left(\alpha_{s}\right)=\alpha_{s} /(12 \pi)+\mathcal{O}\left(\alpha_{s}^{2}\right)$ is the coefficient function containing the dependence on the top quark mass, and is known to order $\alpha_{s}^{4}[7,10]$. The $x \ll 1$ corrections come, in higher order graphs, from integrating two-gluon irreducible amplitudes over the transverse momenta $\mathbf{k}_{1}$ and $\mathbf{k}_{2}$ of the gluons that couple to the Higgs boson. In the full theory, contributions from $\left|\mathbf{k}_{1}\right|,\left|\mathbf{k}_{2}\right| \gg m_{t}$ are suppressed by the top quark loop. In the effective theory, large values of $\left|\mathbf{k}_{1}\right|$ and $\left|\mathbf{k}_{2}\right|$ are allowed all the way up to the kinematic limit $\sqrt{s}$. This results [9] in the perturbative coefficients having stronger singularities in the complex $N$ plane as $N \rightarrow 0$ (with $N$ the moment variable of Eq. (3)). More precisely, double poles $\alpha_{s} / N^{2}$ appear order by order in $\alpha_{s}$.

To determine these contributions, recall the structure of the hard scattering coefficient in the $\overline{\mathrm{MS}}$ subtraction scheme for $N \rightarrow 0$ [9]:

$$
\begin{aligned}
& C_{N}\left(\alpha_{s}, m_{H}^{2} / \mu^{2}\right) \\
& \equiv \int_{0}^{1} d x x^{N-1} C\left(\alpha_{s}, x, m_{H}^{2} / \mu^{2}\right) \\
& =R_{N}^{2}\left(\gamma_{N}\right)\left(m_{H}^{2} / \mu^{2}\right)^{2 \gamma_{N}} h_{N}\left(\gamma_{N}, \gamma_{N}\right) .
\end{aligned}
$$

Here $R_{N}$ is a normalization factor associated with the choice of the $\overline{\mathrm{MS}}$ scheme [11]; $\gamma_{N}$ is a known, universal anomalous dimension, with the perturbation expansion

$\gamma_{N}=\frac{C_{A}}{N} \frac{\alpha_{s}}{\pi}+\mathcal{O}\left(\frac{\alpha_{s}}{N}\right)^{4}$

the function $h_{N}$ is constructed from the matrix element $\hat{\sigma}$ for the off-shell amplitude $g\left(k_{1}\right)+g\left(k_{2}\right) \rightarrow H$ by taking the following integral transform

$$
\begin{aligned}
& h_{N}\left(\gamma_{1}, \gamma_{2}\right) \\
& =\gamma_{1} \gamma_{2} \int \frac{d^{2} \mathbf{k}_{1}}{\pi \mathbf{k}_{1}^{2}}\left(\frac{\mathbf{k}_{1}^{2}}{m_{H}^{2}}\right)^{\gamma_{1}} \int \frac{d^{2} \mathbf{k}_{2}}{\pi \mathbf{k}_{2}^{2}}\left(\frac{\mathbf{k}_{2}^{2}}{m_{H}^{2}}\right)^{\gamma_{2}} \\
& \quad \times \int_{0}^{1} \frac{d x}{x} x^{N} \hat{\sigma}\left(x, \frac{\mathbf{k}_{1}}{m_{H}}, \frac{\mathbf{k}_{2}}{m_{H}}\right) .
\end{aligned}
$$

The matrix element $\hat{\sigma}$, although off-shell, is defined gauge-invariantly by coupling the gluons with eikonal polarizations, as in [9]. It is readily calculated using the effective Lagrangian (2):

$$
\begin{aligned}
\hat{\sigma} & \left(x, \frac{\mathbf{k}_{1}}{m_{H}}, \frac{\mathbf{k}_{2}}{m_{H}}\right) \\
= & \frac{\alpha_{s}^{2} m_{H}^{2} G_{F} \sqrt{2}}{288 \pi} \frac{m_{H}^{4}}{x^{2} \mathbf{k}_{1}^{2} \mathbf{k}_{2}^{2}} \frac{\left(\mathbf{k}_{1} \cdot \mathbf{k}_{2}\right)^{2}}{\left(m_{H}^{2}+\mathbf{k}_{1}^{2}+\mathbf{k}_{2}^{2}\right)^{2}} \\
& \times \frac{1}{x} \delta\left(\frac{1}{x}-1-\frac{\left(\mathbf{k}_{1}+\mathbf{k}_{2}\right)^{2}}{m_{H}^{2}}\right) .
\end{aligned}
$$

Now consider the function $h_{N}$. The $x$ integral in Eq. (5) can be done using the $\delta$-function of Eq. (6):

$$
\begin{aligned}
\int_{0}^{1} & \frac{d x}{x} x^{N} \hat{\sigma} \\
= & \frac{\alpha_{s}^{2} m_{H}^{2} G_{F} \sqrt{2}}{288 \pi} \frac{m_{H}^{4}}{\mathbf{k}_{1}^{2} \mathbf{k}_{2}^{2}} \frac{\left(\mathbf{k}_{1} \cdot \mathbf{k}_{2}\right)^{2}}{\left(m_{H}^{2}+\mathbf{k}_{1}^{2}+\mathbf{k}_{2}^{2}\right)^{2}} \\
& \times \frac{\left(m_{H}^{2}\right)^{N-2}}{\left[m_{H}^{2}+\left(\mathbf{k}_{1}+\mathbf{k}_{2}\right)^{2}\right]^{N-2}} .
\end{aligned}
$$

Notice that when $N \rightarrow 0$ the matrix element is unsuppressed for large $\left|\mathbf{k}_{1}\right|,\left|\mathbf{k}_{2}\right|$. This is a consequence of approximating the Higgs coupling by a local vertex, and is in contrast with the general case of a finite topquark mass [12]. The behavior at large transverse momenta in Eq. (7) causes the function $h$ to have $N=0$ singularities not only through its dependence on the anomalous dimension (4) but also through its explicit 
$N$ dependence. Introducing the notation

$\xi_{1}=\mathbf{k}_{1}^{2} / m_{H}^{2}, \quad \xi_{2}=\mathbf{k}_{2}^{2} / m_{H}^{2}$

and performing the angular integral in Eq. (5) we get

$$
\begin{aligned}
h_{N}\left(\gamma_{1}, \gamma_{2}\right) & \\
= & \frac{\alpha_{s}^{2} m_{H}^{2} G_{F} \sqrt{2}}{288 \pi} \gamma_{1} \gamma_{2} \int_{0}^{\infty} d \xi_{1} \xi_{1}^{\gamma_{1}-1} \int_{0}^{\infty} d \xi_{2} \xi_{2}^{\gamma_{2}-1} \\
& \times\left\{\frac{1}{2\left(1+\xi_{1}+\xi_{2}\right)^{N}}\right. \\
& \times{ }_{2} F_{1}\left(\frac{N-1}{2}, \frac{N-2}{2}, 2, \frac{4 \xi_{1} \xi_{2}}{\left(1+\xi_{1}+\xi_{2}\right)^{2}}\right) \\
& +\frac{\xi_{1} \xi_{2}(N-1)(N-2)}{2\left(1+\xi_{1}+\xi_{2}\right)^{2+N}} \\
& \left.\times{ }_{2} F_{1}\left(\frac{N}{2}, \frac{N+1}{2}, 3, \frac{4 \xi_{1} \xi_{2}}{\left(1+\xi_{1}+\xi_{2}\right)^{2}}\right)\right\},
\end{aligned}
$$

where ${ }_{2} F_{1}$ is the hypergeometric function. The integration region of large $\xi_{1}$ and $\xi_{2}$ in Eq. (9) gives rise to a pole at $\gamma_{1}+\gamma_{2}=N$. This pole is the origin of the double-logarithmic terms.

To approximate $h$ near $N=0$ taking the contribution of this pole into account, we expand the hypergeometric functions in Eq. (9) to the lowest order around $N=0$,

$$
\begin{aligned}
& { }_{2} F_{1}\left(\frac{N-1}{2}, \frac{N-2}{2}, 2, \frac{4 \xi_{1} \xi_{2}}{\left(1+\xi_{1}+\xi_{2}\right)^{2}}\right) \\
& \quad \simeq 1+\frac{\xi_{1} \xi_{2}}{\left(1+\xi_{1}+\xi_{2}\right)^{2}}+\mathcal{O}(N), \\
& (N-1)(N-2)_{2} F_{1}\left(\frac{N}{2}, \frac{N+1}{2}, 3, \frac{4 \xi_{1} \xi_{2}}{\left(1+\xi_{1}+\xi_{2}\right)^{2}}\right) \\
& \quad \simeq 2+\mathcal{O}(N),
\end{aligned}
$$

but retain the $N$ dependence in the factor $\left(1+\xi_{1}+\right.$ $\left.\xi_{2}\right)^{-N}$. The integrations in $\xi_{1}$ and $\xi_{2}$ can now be carried out simply in terms of Euler $\Gamma$ functions:

$$
\begin{aligned}
& h_{N}\left(\gamma_{1}, \gamma_{2}\right) \\
& \simeq \frac{\alpha_{s}^{2} m_{H}^{2} G_{F}}{288 \pi \sqrt{2}} \Gamma\left(1+\gamma_{1}\right) \Gamma\left(1+\gamma_{2}\right) \Gamma\left(N-\gamma_{1}-\gamma_{2}\right) \\
& \quad \times\left[\frac{1}{\Gamma(N)}+3 \gamma_{1} \gamma_{2} \frac{1}{\Gamma(N+2)}\right], \quad N \ll 1 .
\end{aligned}
$$

We can now expand $h$ for small anomalous dimensions, setting $\gamma_{1}=\gamma_{2}=\gamma_{N}$, with $\gamma_{N}$ given in Eq. (4), and determine the leading $N \ll 1$ part of the perturbative coefficients through the second order:

$$
\begin{aligned}
& h_{N}\left(\gamma_{N}, \gamma_{N}\right) \\
& \simeq \frac{\alpha_{s}^{2} m_{H}^{2} G_{F}}{288 \pi \sqrt{2}} \\
& \quad \times\left(1+2 \gamma_{N} \frac{1}{N}+4 \gamma_{N}^{2} \frac{1}{N^{2}}+\cdots\right) \\
& \quad\left(\gamma_{N} \ll 1, N \ll 1\right) .
\end{aligned}
$$

Via Eq. (4), Eq. (13) gives the double-pole terms $\alpha_{s} / N^{2}$ order by order in perturbation theory. These terms are generated by the pole $\left(N-2 \gamma_{N}\right)^{-1}$ in $h$, see Eq. (12).

We are now in a position to find the NNLO correction to the gluon-gluon coefficient for $x \ll 1$. Higher order contributions to $K\left(\alpha_{s}\right)$ [10] in Eq. (2) are subleading for $x \ll 1$. The factor $R_{N}$ in Eq. (3) is a single-logarithmic factor [11] that starts to contribute one order higher than NNLO:

$$
R_{N}=1+\mathcal{O}\left(\alpha_{s} / N\right)^{3} \text {. }
$$

The $\left(m_{H}^{2} / \mu^{2}\right)^{2 \gamma_{N}}$ factor in Eq. (3) is to be expanded for small $\gamma_{N}$, as has been done with $h$. Then, by taking an inverse transform from moment space to $x$-space, we get

$$
\begin{aligned}
C_{g g}\left(\alpha_{s}, x, m_{H}^{2} / \mu^{2}\right) \\
=\frac{\alpha_{s}^{2} m_{H}^{2} G_{F}}{288 \pi \sqrt{2}}\left[\delta(1-x)+\frac{\alpha_{s}}{\pi} C_{A}(-2 \ln x+2 L)\right. \\
\quad+\left(\frac{\alpha_{s}}{\pi}\right)^{2} C_{A}^{2}\left(-\frac{2}{3} \ln ^{3} x+2 \ln ^{2} x L-2 \ln x L^{2}\right) \\
\quad+\cdots] .
\end{aligned}
$$

The first two terms in the square bracket reproduce respectively the leading-order result [3] and the $x \ll 1$ piece of the next-to-leading-order result [4]; the third term determines the $x \ll 1$ pieces of the coefficients $c_{g g}^{(2)}, \bar{c}_{g g}^{(2)}, \overline{\bar{c}}_{g g}^{(2)}$ in Eq. (1).

The quark components of the coefficient also contain double-logarithmic corrections. These can be de- 
termined in an analogous manner. We find

$$
\begin{aligned}
C_{q g}\left(\alpha_{s}, x, m_{H}^{2} / \mu^{2}\right) \\
=\frac{\alpha_{s}^{2} m_{H}^{2} G_{F}}{288 \pi \sqrt{2}}\left[\frac{\alpha_{s}}{\pi} C_{F}(-\ln x+L)+\left(\frac{\alpha_{s}}{\pi}\right)^{2} C_{F} C_{A}\right. \\
\left.\quad \times\left(-\frac{1}{2} \ln ^{3} x+\frac{3}{2} \ln ^{2} x L-\frac{3}{2} \ln x L^{2}\right)+\cdots\right],
\end{aligned}
$$

$$
\begin{aligned}
C_{q \bar{q}}\left(\alpha_{s}, x, m_{H}^{2} / \mu^{2}\right) \\
=\frac{\alpha_{s}^{2} m_{H}^{2} G_{F}}{288 \pi \sqrt{2}}\left(\frac{\alpha_{s}}{\pi}\right)^{2} \\
\quad \times C_{F}^{2}\left(-\frac{1}{3} \ln ^{3} x+\ln ^{2} x L-\ln x L^{2}\right)+\cdots .
\end{aligned}
$$

In summary, perturbative corrections to Higgs production are double-logarithmic for $x \ll 1$ in the $m_{t} \rightarrow$ $\infty$ effective theory, while single-logarithmic in the full theory. We have given simple formulas that incorporate all the double logarithms to any order in $\alpha_{s}$ in the $m_{t} \rightarrow \infty$ case. The formulas also contain subleading $N \rightarrow 0$ terms, mostly of kinematical origin, which may be relevant for matching the $x \ll 1$ contributions with expansions around the soft limit $x \rightarrow 1[1,5,6]$. For observables sensitive to the $x \ll 1$ region, it will be relevant to include finite top-mass corrections.

\section{References}

[1] R.V. Harlander, W.B. Kilgore, hep-ph/0201206.

[2] M.A. Shifman, A.I. Vainshtein, M.B. Voloshin, V.I. Zakharov, Sov. J. Nucl. Phys. 30 (1979) 711;

J. Ellis, M.K. Gaillard, D. Nanopoulos, Nucl. Phys. B 106 (1976) 292

[3] H. Georgi, S. Glashow, M. Machacek, D. Nanopoulos, Phys. Rev. Lett. 40 (1978) 692.

[4] A. Djouadi, M. Spira, P.M. Zerwas, Phys. Lett. B 264 (1991) 440 S. Dawson, Nucl. Phys. B 359 (1991) 283.

[5] S. Catani, D. de Florian, M. Grazzini, JHEP 0201 (2002) 015.

[6] S. Catani, D. de Florian, M. Grazzini, JHEP 0105 (2001) 025; R.V. Harlander, W.B. Kilgore, Phys. Rev. D 64 (2001) 013015.

[7] M. Krämer, E. Laenen, M. Spira, Nucl. Phys. B 511 (1998) 523.

[8] R.V. Harlander, Phys. Lett. B 492 (2000) 74.

[9] S. Catani, M. Ciafaloni, F. Hautmann, Phys. Lett. B 242 (1990) 97;

S. Catani, M. Ciafaloni, F. Hautmann, Nucl. Phys. B 366 (1991) 135.

[10] K.G. Chetyrkin, B.A. Kniehl, M. Steinhauser, Nucl. Phys. B 510 (1998) 61;

K.G. Chetyrkin, B.A. Kniehl, M. Steinhauser, Phys. Rev. Lett. 79 (1997) 353.

[11] S. Catani, F. Hautmann, Nucl. Phys. B 427 (1994) 475; S. Catani, M. Ciafaloni, F. Hautmann, Phys. Lett. B 307 (1993) 147.

[12] M. Spira, A. Djouadi, D. Graudenz, P.M. Zerwas, Nucl. Phys. B 453 (1995) 17. 